\title{
Muscle Activation Analysis of Flatfoot According to the Slope of a Treadmill
}

\author{
Myoung-Kwon Kim, PT, PhD ${ }^{1)}$, Chang-Ryeol Lee, PT, PhD²) \\ 1) Department of Physical Therapy, Youngsan University: San 150 Joonam-dong, Yangsan, \\ Kyeongsangnam-do, Republic of Korea. TEL: +82 55-380-9367, FAX: +82 55-380-9305, E-mail: \\ skybird-98@hanmail.net \\ 2) Department of Physical Therapy, Korea Nazarene University
}

\begin{abstract}
Purpose] The purpose of this study was to determine the difference between the flatfoot and normal foot on slopes using electromyography. [Subjects] This study was conducted on 30 adults having normal feet $(\mathrm{N}=15)$ and flatfeet $(\mathrm{N}=15)$ from who were 21 to 30 years old and no neurological history or gait problems. [Methods] A treadmill (AC5000M, SCIFIT, UK) was used to analyze kinematic features during walking, using slopes of -10 , 0 , and $10 \%$ and normal gait velocities. A surface electromyogram (TeleMyo 2400T, Noraxon Co., USA) was used to measure muscle activity changes. [Results] Muscle activity of the flatfoot was significantly different at most muscles, and muscle activity in the normal foot was significantly different in the vastus medialis, vastus lateralis, tibialis anterior, peroneus longus, medial gastrocnemius, and lateral gastrocnemius. Comparison of muscle activity between the flatfoot and normal foot showed significant differences in the tibialis anterior and abductor hallucis muscle. [Conclusion] In the gait of people with flatfoot while walking on slopes, muscle activation of the tibialis anterior is higher than for normal feet and muscle activation of the abductor hallucis muscle is relatively lower than for normal feet in the gait while walking on slopes. This is due to lack of the ability to absorb shock on the ground as a result of the weakned function of the medial longitudinal arch.
\end{abstract}

Key words: Gait, Slope, Electromyography

(This article was submitted Jun. 29, 2012, and was accepted Aug. 13, 2012)

\section{INTRODUCTION}

Flatfoot is a congenital or acquired disease and shows features such as medial rotation of the talus, decrease of the medial longitudinal arch, supination, and abduction of the forefoot ${ }^{1)}$. Mechanical causes of flatfoot have been explained by many researchers. Firstly, flatfoot can be generated because of dysfunction of the posterior tibial tendon. This increases the load on the ligament supporting the joint of the first metatarsal bone and medial longitudinal arch. This reduces the height of the medial longitudinal arch, and it gradually becomes flat ${ }^{2,3)}$. It can also be generated by injury, such as to the spring ligament ${ }^{4}$ and plantar fascia ${ }^{5)}$, which supports a stability of the medial longitudinal arch. Overweight also raises the incidence of flatfoot ${ }^{6}$. Recently, there have been many studies relating to gait. Flatfoot has been studied with basic technology using various complex methods ${ }^{7}$. The study of flatfoot is constantly evolving, developing from visual quantitative examination to mathematical analysis and modeling through to more complex measurement methods with the development of computer and electronics technologies ${ }^{8}$. The causes and abnormal features of patients with a pathological gait need to be determined through analysis and accumulation of analysis results. We then need to identify objective gait types for normal adults. Therefore, this study was conducted to determine the difference between the flatfoot and the normal foot by gait velocity and slopes using electromyography.

\section{SUBJECTS AND METHODS}

This study was conducted on 30 adults having normal feet $(\mathrm{N}=15)$ and flatfeet $(\mathrm{N}=15)$ who were 21 to 30 years old and had no neurological history or gait problems. A treadmill (AC5000M, SCIFIT, UK) was used to analyze kinematic features during walking, using slopes of $-10,0$, and $10 \%$ with normal gait velocities. A surface electromyogram (TeleMyo 2400T, Noraxon Co., USA) was used to measure muscle activity changes. Flatfoot was diagnosed by postural analysis with a foot pressure analysis device (GPS400, Redbalance, Italy). Flatfoot was evaluated using Strake's line and Marie's line as explained by Clarke ${ }^{9}$. Strake's line passes through the medial border of the forefoot and hindfoot, and Marie's line passes through the center of the $3^{\text {rd }}$ metatarsal bones and hindfoot. Diagnosis of flatfoot was made based on Strake's line and Marie's line. In the normal foot, the line of the medial arch passes lateral to Marie's line. In the flatfoot, the line of the medial arch passes medial to Marie's line. In this study, the laboratory environment was made comfortable and had a pleasant indoor temperature, and we obtained the consent of the subjects for participation in the study. A treadmill (AC5000M, SCIFIT, UK) was used 
for walking, and $-10,0,10 \%$ slopes were used based on the study of Leroux et al. Gait velocity for the subjects was $1.20 \mathrm{~m} / \mathrm{s}$ which represents the normal gait velocity of young people in their 20s in Korea. Most of the subjects walked on the treadmill set at the three different slopes for 5 minutes. The subjects walked barefoot and looked towards the front while walking. Each subject walked for one minute to ensure a natural gait before the experiment. We collected the data by wireless surface electromyography (TeleMyo 2400T, Noraxon co., USA). The active electrode was composed of two stainless steel pads; the diameter of the electrode was $11.4 \mathrm{~mm}$, and the distance of the electrode was $20 \mathrm{~mm}$. The signal was stored and analyzed using the software of the TeleMyo 2400T system (Noraxon U.S.A Inc.). The sampling rate for the electromyography signal was set to $1000 \mathrm{~Hz}$, the bandwidth was set to $20-450 \mathrm{~Hz}$, and the notch filter was set to $60 \mathrm{~Hz}$. Electromyography (EMG) was performed after depilating the electrode attachment areas with a razor, removing the horny layer with sand paper, and cleansing with an alcohol swab. To measure muscle activations in the lower extremity during walking, electrodes were attached to the abductor hallucis muscle, tibialis anterior muscle, peroneus longus muscle, medial gastrocnemius muscle, lateral gastrocnemius muscle, vastus medialis, vastus lateralis, and biceps femoris muscle. The surface electrodes used were composed of three electrodes. The frequency range of the EMG signal was between 20 and $500 \mathrm{~Hz}$, and the sampling frequency was $1024 \mathrm{~Hz}$. We normalized the signal of each muscle with \%MVC which compare with RMS and maximal voluntary isometric contraction. Analysis of homogeneous data for general characteristics of the subjects, age, height, and weight was conducted with the independent $t$-test. Measured data were analyzed by repeated measures ANOVA in SPSS for Windows (version 17.0). The differences between groups according to slopes were measured with the independent $t$-test. Statistical significance was accepted for $p$ values of $<0.05$.

\section{RESULTS}

The general characteristic of the subjects are showed in Table 1. Muscle activity for the flatfoot was significantly different in most muscles, and muscle activity for the normal foot was significantly different in the vastus medialis, vastus lateralis, tibialis anterior, peroneus longus, medial gastrocnemius and lateral gastrocnemius $(\mathrm{p}<0.05)$. Comparison of muscle activity between the flatfoot and normal foot showed significant difference in the tibialis anterior and abductor hallucis muscle $(\mathrm{p}<0.05)$ (Table 2$)$.

\section{DISCUSSION}

Electromyography can evaluate nerve root injury and can be used as a tool for evaluating nerve conduction velocity, and so it can be used to confirm the position of a peripheral nerve injury ${ }^{10)}$. It can also be useful when we analyze a limb segment kinematically and perform clinical analysis of functional activities such as walking, stair climbing and so on. Ground reaction force of a lower extremity supporting
Table 1. General characteristics of each group

\begin{tabular}{lcc}
\hline & EG $(\mathrm{n}=15)$ & $\mathrm{CC}(\mathrm{n}=15)$ \\
\hline $\begin{array}{l}\text { Number of individuals } \\
\text { (male / female) }\end{array}$ & $5 / 10$ & $7 / 8$ \\
Age (years) & $21.7 \pm 1.5$ & $22.0 \pm 2.1$ \\
Height $(\mathrm{cm})$ & $165.1 \pm 0.7$ & $164.2 \pm 6.5$ \\
Body weight $(\mathrm{kg})$ & $55.8 \pm 6.2$ & $54.2 \pm 4.2$ \\
Foot length $(\mathrm{mm})$ & $255.4 \pm 10.7$ & $252.8 \pm 5.1$ \\
Ankle width $(\mathrm{cm})$ & $7.89 \pm 0.37$ & $7.60 \pm 0.53$ \\
Navicular drop $(\mathrm{mm}) *$ & $11.6 \pm 0.3$ & $6.0 \pm 0.1$ \\
\hline (Mean $+\mathrm{SD}) * \mathrm{p}<0.05, \mathrm{EG} \cdot$ Experimental group, $\mathrm{CC} \cdot$ Control group
\end{tabular}

weight and flexion angle of the knee joint could control the amount of power applied to the body during walking. But lower extremities could be injured when it cannot be controlled well.

The ratio of load and power applied to the lower extremity could affect the amount of stress in tissues, and injury could result from repetitive stress. Lower extremities could also be injuried more easily in a sloped environment than on ground ${ }^{11)}$. The vastus medialis and tibialis anterior muscle showed higher muscle activation in the flatfoot group than in the normal foot group on the slopes. Vastus medialis oblique muscle has an important meaning as stability and direction of patella ${ }^{12)}$. Cheung ${ }^{5)}$ confirmed that loading in the vastus medialis is different according to the foot position in patients with patellofemoral pain syndrome. It was also identified that in the case of the flatfoot, the activity of the tibialis anterior muscle increased during the stance phase of the gait cycle compared with the normal foot as a way to absorb the impact delivered to the foot while the pronation of the subtalar joint progresses. The tibialis anterior muscle is an agonist of the ankle joint dorsiflexion and plays an important role in eccentric control to achieve appropriate toe clearance during walking. The tibialis anterior muscle shows the maximum muscle activity in the initial swing phase and terminal swing phase and responds sensitively to walking speeds during the terminal swing phase. The tibialis anterior muscle serves the role of a dorsiflexor during the initial swing phase in order to achieve toe clearance and performs eccentric control during the terminal swing phase for anticipatory responses to the loads imposed during the initial stance phase ${ }^{13)}$. In a study that compared the activity of the tibialis anterior muscle during walking on a flat land with that during walking on sloped roads ${ }^{14)}$, Lay et al. ${ }^{15)}$ could not find any significant difference. However, in that study, the activity of the tibialis anterior muscle was shown to be higher on descending sloped roads or ascending sloped roads compared with roads on flat land and in particular, higher muscle activity was shown in the case of flat feet compared to normal feet.

Relatively higher muscle activation of the tibialis anterior muscle is caused to compensate for shock absorption of the foot. The abductor hallucis muscle is positioned below the medial longitudinal arch of the foot and inserted from the calcaneus into the sesamoid of the distal phalanx. Its function is to provide dynamic stability to the medial longi- 
Table 2. Comparison according to velocity on various slopes

\begin{tabular}{llccc}
\hline & Group & $-10 \%$ & $0 \%$ & $10 \%$ \\
\hline \multirow{2}{*}{ Rectus femoris } & EG* & $22.5 \pm 3.7$ & $21.6 \pm 3.2$ & $24.1 \pm 3.2$ \\
& CC & $23.2 \pm 1.5$ & $21.6 \pm 1.0$ & $23.7 \pm 3.9$ \\
\hline \multirow{2}{*}{ Vastus medialis } & EG* $^{*} 26.2 \pm 6.0$ & $21.8 \pm 3.9$ & $25.1 \pm 5.8$ \\
& CC* $^{*}$ & $25.8 \pm 1.2$ & $21.6 \pm 1.1$ & $24.2 \pm 1.4$ \\
\hline \multirow{2}{*}{ Vastus lateralis } & $\mathrm{EG}^{*}$ & $22.1 \pm 2.5$ & $20.8 \pm 1.0$ & $23.8 \pm 1.6$ \\
& $\mathrm{CC}^{*}$ & $23.0 \pm 1.0$ & $21.8 \pm 1.3$ & $24.3 \pm 1.9$ \\
\hline \multirow{2}{*}{ Tibialis anterior } & $\mathrm{EG}^{*}$ & $23.8 \pm 1.2^{\mathrm{a}}$ & $22.0 \pm 0.9$ & $24.8 \pm 1.4$ \\
& $\mathrm{CC}^{*}$ & $22.1 \pm 0.9^{\mathrm{a}}$ & $20.8 \pm 1.8$ & $25.2 \pm 2.1$ \\
\hline \multirow{2}{*}{ Peroneus longus } & $\mathrm{EG}^{*}$ & $20.7 \pm 1.4$ & $22.1 \pm 1.0$ & $27.0 \pm 2.0$ \\
& $\mathrm{CC}^{*}$ & $22.0 \pm 1.8$ & $23.3 \pm 2.1$ & $27.6 \pm 3.4$ \\
\hline \multirow{2}{*}{ Medial gastrocnemius } & $\mathrm{EG}^{*}$ & $32.6 \pm 4.1$ & $33.5 \pm 4.5$ & $34.2 \pm 4.6$ \\
& $\mathrm{CC}^{*}$ & $34.2 \pm 1.8$ & $33.6 \pm 1.3$ & $34.6 \pm 1.3$ \\
\hline \multirow{2}{*}{ Lateral gastrocnemius } & $\mathrm{EG}^{*}$ & $34.6 \pm 1.4$ & $33.1 \pm 1.4$ & $34.4 \pm 1.6$ \\
& $\mathrm{CC}^{*}$ & $34.4 \pm 1.4$ & $33.1 \pm 0.6$ & $34.1 \pm 1.3$ \\
\hline \multirow{2}{*}{ Abductor hallucis } & $\mathrm{EG}^{*}$ & $14.6 \pm 1.5^{\mathrm{a}}$ & $16.0 \pm 4.0$ & $17.3 \pm 4.0^{\mathrm{a}}$ \\
& $\mathrm{CC}^{\mathrm{a}}$ & $17.4 \pm 4.3^{\mathrm{a}}$ & $18.9 \pm 6.4$ & $21.4 \pm 5.0^{\mathrm{a}}$ \\
\hline
\end{tabular}

$(\% \mathrm{MVC}) * \mathrm{p}<0.05$, EG: Experimental group, $\mathrm{CC}$ : Control group. ${ }^{\mathrm{a}}$ significant difference $(\mathrm{p}<0.05)$ between the groups according the slope

tudinal $\operatorname{arch}^{16)}$. In this study, changes in the activity of the abductor hallucis muscle of the flatfoot in relation to changes in the degree of slope were not large compared with those of the normal foot. This result can be seen as meaning that the abductor hallucis muscle does not properly perform the function of a muscle for the stability of the medial longitudinal arch. By blocking the tibia nerve that controls the abductor hallucis muscle ${ }^{17)}$ and inducing fatigue, it was identified that the abductor hallucis muscle affected the drop of the navicular bone ${ }^{18)}$. Metatarsophalangeal joint bending exercises have been shown to be helpful for strengthening intrinsic muscles such as the abductor hallucis muscle ${ }^{19,20)}$.

\section{ACKNOWLEDGEMENT}

This study was financially supported by the research fund of Korea Nazarene University in 2013.

\section{REFERENCES}

1) Arangio GA, Reinert KL, Salathe EP: A biomechanical model of the effect of subtalar arthroereisis on the adult flexible flat foot. Clin Biomech (Bristol, Avon), 2004, 19: 847-852. [Medline] [CrossRef]

2) Deland JT, de Asla RJ, Sung IH, et al.: Posterior tibial tendon insufficiency: which ligaments are involved? Foot Ankle Int, 2005, 26: 427-435. [Medline]

3) Arangio GA, Chen C, Salathe EP: Effect of varying arch height with and without the plantar fascia on the mechanical properties of the foot. Foot Ankle Int, 1998, 19: 705-709. [Medline]

4) Lloyd DG, Besier TF: An EMG-driven musculoskeletal model to estimate muscle forces and knee joint moments in vivo. J Biomech, 2003, 36: 765-776. [Medline] [CrossRef]

5) Cheung RT, NG GY: Motion control shoe affects temporal activity of quadriceps in runners. Br J Sports Med, 2009, 43: 943-947. [Medline]
[CrossRef]

6) Pfeiffer M, Kotz R, Ledl T, et al.: Prevalence of flat foot in preschool-aged children. Pediatrics, 2006, 118: 634-639. [Medline] [CrossRef]

7) Perry J, Burnfield J: Gait analysis; normal and pathological function, SLACK, 2010.

8) Razeghi M, Batt ME: Foot type classification: a critical review of curren methods. Gait Posture, 2002, 15: 282-291. [Medline] [CrossRef]

9) Clarke HH: Application of measurement to health and physical education, 5th ed., 1976

10) Hogrel JY: Clinical applications of surface electromyography in neuromuscular disorders. Neurophysiol Clin, 2005, 35: 59-71. [Medline] [CrossRef]

11) Hargrave MD, Carcia CR, Gansneder BM, et al.: Subtalar pronation does not influence impact forces or rate of loading during a single-leg landing. J Athl Train, 2003, 38: 18-23. [Medline]

12) Neumann DA: Kinesiology of the musculoskeletal system: Foundations for physical rehabilitation. Mosby, 2002.

13) Lamont EV, Zehr EP: Task-specific modulation of cutaneous reflexes expressed at functionally relevant gait cycle phases during level and incline walking and stair climbing. Exp Brain Res, 2006, 173: 185-192. [Medline] [CrossRef]

14) den Otter AR., Geurts AC, Mulder T, et al.: Speed related changes in muscle activity from normal to very slow walking speeds. Gait Posture, 2004, 19: 270-278. [Medline] [CrossRef]

15) Lay AN, Hass CJ, Nichols TR, et al.: The effects of sloped surfaces on locomotion; an electromyographic analysis. J Biomech, 2007, 40: 1276-1285. [Medline] [CrossRef]

16) Wong YS: Influence of the abductor hallucis muscle on the medial arch of the foot: a kinematic and anatomical cadaver study. Foot Ankle Int, 2007, 28: 617-620. [Medline] [CrossRef]

17) Fiolkowski P, Brunt $D$, Bishop $M$, et al.: Intrinsic pedal musculature support of the medial longitudinal arch: an electromyography study. J Foot Ankle Surg, 2003, 42: 327-333. [Medline] [CrossRef]

18) Headlee DL, Leonard JL, Hart JM, et al.: Fatigue of the plantar intrinsic foot muscles increases navicular drop. J Electromyogr Kinesiol, 2008, 18: 420-425. [Medline] [CrossRef]

19) Greenman P: Principles of manual medicine. 2nd ed. Baltimore; Lippincott, Williams \& Wilkins, 2003.

20) Rothermel SA, Hale SA, Hertel J, et al.: Effect of active foot positioning on the outcome of a balance training program. Phys Ther Sport, 2004, 5: 98-103. 